\title{
Temporal decorrelations in compressible isotropic turbulence
}

\author{
Dong Li, Xing Zhang, and Guowei He* \\ LNM, Institute of Mechanics, Chinese Academy of Sciences, Beijing 100190, China \\ (Received 27 February 2013; published 14 August 2013)
}

\begin{abstract}
Temporal decorrelations in compressible isotropic turbulence are studied using the space-time correlation theory and direct numerical simulation. A swept-wave model is developed for dilatational components, while the classic random sweeping model is proposed for solenoidal components. The swept-wave model shows that the temporal decorrelations in dilatational fluctuations are dominated by two physical processes: random sweeping and wave propagation. These models are supported by the direct numerical simulation of compressible isotropic turbulence, in the sense that all curves of normalized time correlations for different wave numbers collapse into a single one using the normalized time separations. The swept-wave model is further extended to account for a constant mean velocity.
\end{abstract}

DOI: 10.1103/PhysRevE.88.021001

PACS number(s): 47.27.eb, 47.27.Gs, 47.40.-x

A milestone in isotropic and homogeneous turbulence is the random sweeping hypothesis [1,2]. The random sweeping hypothesis proposes a temporal decorrelation process in incompressible isotropic turbulence and a simple model for space-time correlations of velocity fluctuations [1]. These results are examined theoretically [3-5] and verified experimentally [6] and numerically [7,8]. The space-time correlation models are used to predict the scalings of wave number or frequency energy spectra in turbulent flows [9-12]. The decorrelation processes are also relevant to the non-Gaussian statistics [13] and intermittency [14]. Their further applications can be found in turbulence generated noise [15]. The recently increasing studies on compressible isotropic turbulence raise such a question on the effects of compressibility on decorrelation processes [16-18]. In this Rapid Communication, we will study the decorrelation processes in compressible isotropic turbulence and propose a model for space-time correlations of dilatational components.

A compressible turbulence is associated with two characteristic velocities: fluid velocity and sound speed, whereas an incompressible one is only associated with fluid velocity. Therefore, the decorrelation processes in compressible turbulence are very different from the incompressible one. A space-time correlation is the essential quantity to measure the decorrelation processes in turbulent flows. Three typical models exist for space-time correlations in turbulence theory. The first one is, as stated above, the random sweeping model for incompressible turbulence [1]. We will show that it cannot characterize the dilatational components in compressible turbulence. The second one is the Taylor frozen flow model [19]. It has been shown that this model is not a good approximation for dilatational components [20]. The third one is the linear wave propagation model [20]. This model has been used for dilatational components when compressible turbulence has a dominating mean velocity. However, it does not decrease with increasing temporal separation, which violates the nature of correlation functions.

In this Rapid Communication, we will develop a space-time correlation model for compressible isotropic turbulence. This

\footnotetext{
*hgw@lnm.imech.ac.cn
}

is achieved by the Helmholtz decomposition: a velocity field can be split into the solenoidal and dilatational components. A swept-wave model will be developed for the dilatational components, while the solenoidal components are expected to follow the random sweeping model. The swept-wave model will be numerically validated and further used to elucidate the decorrelation process in compressible turbulence.

We consider compressible and isotropic turbulence with periodic boundary conditions. In this case, the Helmholtz decomposition for velocity fields can be made as follows [10]:

$$
\mathbf{u}=\mathbf{u}^{s}+\mathbf{u}^{d},
$$

where $\mathbf{u}^{s}$ and $\mathbf{u}^{d}$ are the solenoidal (i.e., incompressible) and dilatational components, respectively. The harmonic component is taken to be zero. We will investigate the temporal decorrelations of solenoidal and dilatational components. The temporal decorrelations can be measured by the space-time correlations of velocity fluctuations,

$$
R(r, \tau)=\left\langle u_{\ell}(\mathbf{x}, t) u_{\ell}(\mathbf{x}+\mathbf{r}, t+\tau)\right\rangle,
$$

or its equivalent forms in Fourier space,

$$
\hat{R}(k, \tau)=\left\langle\hat{u}_{\ell}(\mathbf{k}, t) \hat{u}_{\ell}(-\mathbf{k}, t+\tau)\right\rangle .
$$

Here, $r$ and $k$ are the magnitudes of separation vector $\mathbf{r}$ and wave-number vector $\mathbf{k}$. The similar quantities $R^{s}$ and $R^{d}$ can be defined for the solenoidal and dilatational components $\mathbf{u}^{s}$ and $\mathbf{u}^{d}$, respectively. $u_{\ell}, u_{\ell}^{s}$, and $u_{\ell}^{d}$ denote the components of $\mathbf{u}, \mathbf{u}^{s}$, and $\mathbf{u}^{d}$ along any axis.

We propose that a solenoidal component follows the same decorrelation process as the random sweeping process for incompressible isotropic turbulence: small eddies are randomly convected or swept by energy-contained eddies, where the contribution of dilatational components to the energy-contained eddies is comparably small. The random sweeping process can be described by a simple idealized convection equation [1]:

$$
\left(\frac{\partial}{\partial t}+v_{j} \frac{\partial}{\partial x_{j}}\right) u_{\ell}^{s}=0,
$$

where $\mathbf{v}=\left(v_{1}, v_{2}, v_{3}\right)$ is constant in space and time and has an isotropic and Gaussian distribution over an ensemble of realizations. $V=|\mathbf{v}| / \sqrt{3}$ is the rms of component velocity. Its 
solution in the Fourier space is given by

$$
\hat{u}_{\ell}^{s}(\mathbf{k}, t)=\hat{u}_{\ell}^{s}(\mathbf{k}, 0) \exp [-i(\mathbf{k} \cdot \mathbf{v}) t] .
$$

The normalized time correlation of the Fourier mode is formulated as

$$
c^{s}(k, \tau)=\frac{\hat{R}^{s}(k, \tau)}{\hat{R}^{s}(k, 0)}=\exp \left(-\frac{1}{2} V^{2} k^{2} \tau^{2}\right) .
$$

A dilatational component in compressible isotropic turbulence propagates at the speed of sound relative to moving fluids. This implies that the dilatational fluctuations are swept by the energy-contained eddies. Therefore, the temporal decorrelations in dilatational components are governed by two dynamic processes: random sweeping and wave propagation. The well-known linear wave propagation model [20] only includes the wave propagation process. In order to account for the random sweeping effect, we introduce a new term, $\mathbf{v} \cdot \nabla$, into the linear wave propagation equation and propose the governing equation for dilatational fluctuations as follows:

$$
\left(\frac{D^{2}}{D t^{2}}-\bar{a}^{2} \nabla^{2}\right) u_{\ell}^{d}=0
$$

where $\bar{a}$ is the mean speed of sound and

$$
\frac{D}{D t}=\left(\frac{\partial}{\partial t}+\mathbf{v} \cdot \nabla\right)=\left(\frac{\partial}{\partial t}+v_{j} \frac{\partial}{\partial x_{j}}\right) .
$$

Here, $\mathbf{v}$ is the same flow field as in Eq. (4). The new term $\mathbf{v} \cdot \boldsymbol{\nabla}$ in Eq. (7) represents the random sweeping effect, which is absent in the linear wave propagation model [20]. The solution of Eq. (7) in Fourier space is given by

$$
\begin{aligned}
\hat{u}_{\ell}^{d}(\mathbf{k}, t)= & \hat{u}_{\ell}^{d+}(\mathbf{k}, 0) \exp [-i(\mathbf{k} \cdot \mathbf{v}) t-i k \bar{a} t] \\
& +\hat{u}_{\ell}^{d-}(\mathbf{k}, 0) \exp [-i(\mathbf{k} \cdot \mathbf{v}) t+i k \bar{a} t]
\end{aligned}
$$

where $\hat{u}_{\ell}^{d+}$ and $\hat{u}_{\ell}^{d-}$ are the Fourier coefficients. The time correlation of the Fourier mode is calculated as follows:

$$
\begin{aligned}
\hat{R}^{d}(k, \tau)= & \left\langle\hat{u}_{\ell}^{d}(\mathbf{k}, t) \hat{u}_{\ell}^{d}(-\mathbf{k}, t+\tau)\right\rangle \\
= & \left\langle\hat{u}_{\ell}^{d+}(\mathbf{k}, 0) \hat{u}_{\ell}^{d+}(-\mathbf{k}, 0)\right\rangle\langle\exp [i(\mathbf{k} \cdot \mathbf{v}) \tau+i k \bar{a} \tau]\rangle \\
& +\left\langle\hat{u}_{\ell}^{d-}(\mathbf{k}, 0) \hat{u}_{\ell}^{d-}(-\mathbf{k}, 0)\right\rangle\langle\exp [i(\mathbf{k} \cdot \mathbf{v}) \tau-i k \bar{a} \tau]\rangle .
\end{aligned}
$$

Here, the mode correlation $\hat{R}^{d}(k, 0)$ is denoted by

$$
\frac{1}{2} \hat{R}^{d}(k, 0)=\left\langle\hat{u}_{\ell}^{d+}(\mathbf{k}, 0) \hat{u}_{\ell}^{d+}(-\mathbf{k}, 0)\right\rangle=\left\langle\hat{u}_{\ell}^{d-}(\mathbf{k}, 0) \hat{u}_{\ell}^{d-}(-\mathbf{k}, 0)\right\rangle .
$$

Therefore, the normalized correlation function can be expressed as

$$
\begin{aligned}
c^{d}(k, \tau) & =\frac{\hat{R}^{d}(k, \tau)}{\hat{R}^{d}(k, 0)} \\
& =\cos (k \bar{a} \tau) \exp \left(-\frac{1}{2} V^{2} k^{2} \tau^{2}\right) .
\end{aligned}
$$

The swept-wave model (11) contains two factors: a wave function and an exponential function. The first factor represents the wave propagation process, and the second one represents the random sweeping effect. If the sweeping velocity is zero, it becomes the linear wave propagation model. In fact, the linear wave propagation model in compressible isotropic turbulence is simplified as $\cos (k \bar{a} \tau)$, which is the
TABLE I. Runs and parameters.

\begin{tabular}{lcccc}
\hline \hline Run & $\operatorname{Re}_{\lambda}$ & $M_{t}$ & $V$ & $\bar{a}$ \\
\hline $256 \mathrm{~F} 1$ & 84 & 0.42 & 0.24 & 0.98 \\
$256 \mathrm{~F} 2$ & 78 & 0.33 & 0.23 & 1.2 \\
\hline \hline
\end{tabular}

inverse Fourier transformation of Eq. (19) in [20]. This cosine function does not decay to zero as time separation increases.

We will use the data from direct numerical simulations (DNS) of the compressible isotropic turbulence in a cubic box to validate the swept-wave model. For this purpose, the Navier-Stokes equations with periodic conditions are solved using the same method as in Ref. [21], where an optimized sixth-order compact method and a fourth-order two-step low dissipation and dispersion Runge-Kutta scheme [22] are used. The fluid is assumed to be a perfect gas with specific heat ratio $\gamma=1.4$ and Prandtl number $\operatorname{Pr}=0.7$. An external force $\mathbf{f}(\mathbf{x}, t)[23]$ and a uniform cooling in space are introduced into the Navier-Stokes equations to maintain statistically stationary states. The external force is obtained from its Fourier mode $\hat{f}_{i}=\left(\delta_{i j}-k_{i} k_{j} / k^{2}\right) \hat{g}_{j}$ for $1 \leqslant k \leqslant 3$, where $\hat{g}_{j}$ is generated by independent Ornstein-Uhlenbeck processes [24]. Two cases at $256^{3}$ grid resolution are performed with the resulting parameters listed in Table I, where the sweeping velocities $V$ are very close but the mean sound speeds $\bar{a}$ are different. $\operatorname{Re}_{\lambda}$ is the Taylor microscale Reynolds number, and $M_{t}=\sqrt{3} V / \bar{a}$ is the turbulent Mach number. A total of 441 flow fields are taken to calculate time correlations. We find that the dilatational components need more time to reach the statistically stationary states. The ensemble averaging in Eq. (3) is taken over the wave-number shell $k=|\mathbf{k}|$ and time $t$ [25]. In Figs. 1-4, we will only present the results from DNS $256 \mathrm{~F} 2$.

Figure 1 shows the correlation coefficients of solenoidal modes for wave numbers $k=15,30, \ldots, 75$. Obviously, the solenoidal modes decorrelate more quickly at larger wave numbers than at small wave numbers. These results in Fig. 1 are all plotted together in Fig. 2, with the horizontal axis defined by the normalized time scale $\tau_{V}=V k \tau$. This normalization causes excellent collapse of the correlation coefficients. The collapse on the normalized time scale $\tau_{V}$ supports the random sweeping hypothesis for the solenoidal components.

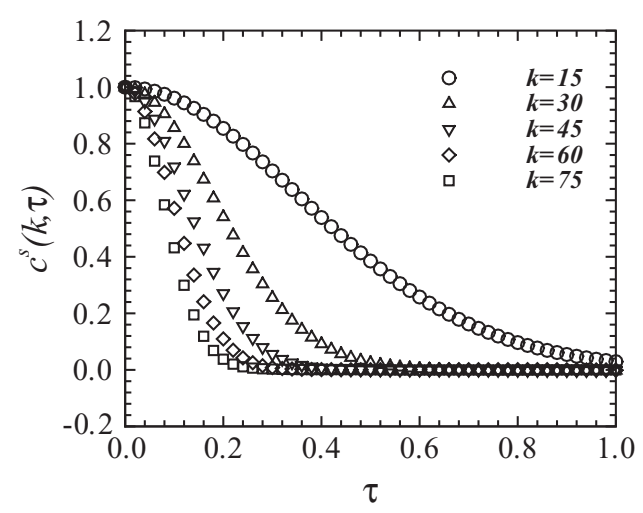

FIG. 1. Normalized time correlations of solenoidal components vs time separations for $k=15,30,45,60,75$. 


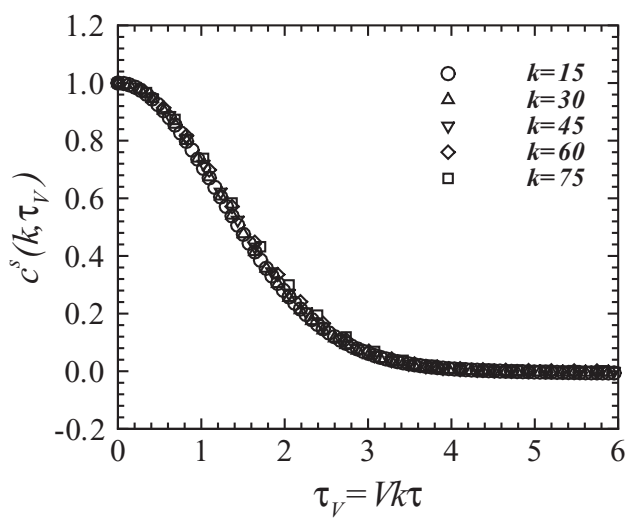

FIG. 2. Normalized time correlations of solenoidal components vs normalized time separations $\tau_{V}=V k \tau$ for $k=15,30,45,60,75$.

Figure 3 plots the normalized time correlations for dilatational components. It is observed that the time correlations of dilatational components decay with oscillations. This is very different from solenoidal components where the time correlations decay without any oscillation. These oscillatory decays confirm that temporal decorrelations in dilatational components are mainly determined by both random sweeping and wave propagation.

Figure 4 presents the normalized correlation functions versus the normalized time scale $\tau_{V}$. The normalization leads to the virtual collapse of all curves. The collapsed curves verify the swept-wave model. We further plot the exponential function $\exp \left(-0.5 \tau_{V}^{2}\right)$. The exponential function acts as an envelope of the collapsed curves for dilatational components. This is in agreement with the swept-wave model.

The swept-wave model can be expressed as $c^{d}\left(k, \tau_{a}\right)=$ $\cos \left(\tau_{a}\right) \exp \left[-\tau_{a}^{2} M_{t}^{2} / 6\right]$ in terms of the scale-similarity variable $\tau_{a}=\bar{a} k \tau$. We further introduce a rescaled correlation function $c^{d *}\left(k, \tau_{a}\right)=c^{d}\left(k, \tau_{a}\right) \exp \left[-\tau_{a}^{2}\left(M_{t 1}^{2}-M_{t}^{2}\right) / 6\right]$, where $M_{t 1}$ is the turbulent Mach number of DNS 256F1. Therefore, $c^{d *}\left(k, \tau_{a}\right)=\cos \left(\tau_{a}\right) \exp \left[-\tau_{a}^{2} M_{t 1}^{2} / 6\right]$ holds for different turbulent Mach numbers. In other words, the rescaled correlation $c^{d *}\left(k, \tau_{a}\right)$ is invariant for both DNS $256 \mathrm{~F} 1$ and $256 \mathrm{~F} 2$. Figure 5 plots the rescaled correlation functions $c^{d *}\left(15, \tau_{a}\right)$ from DNS $256 \mathrm{~F} 1$ and $256 \mathrm{~F} 2$. It is found that those two curves are well collapsed. Those results again support the swept-wave model.

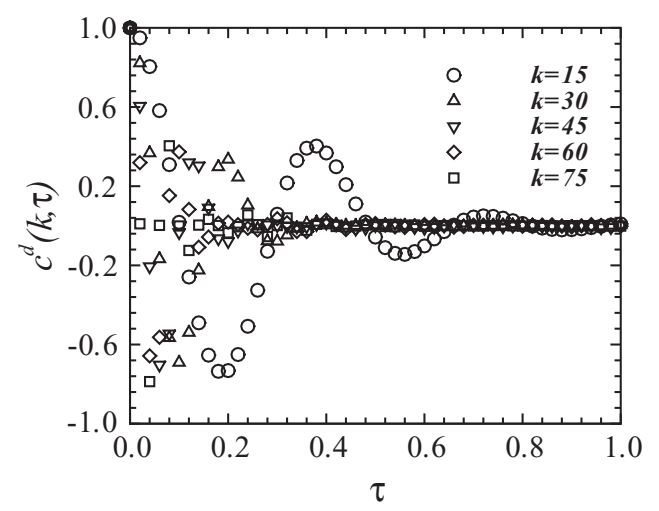

FIG. 3. Normalized time correlations of dilatational components vs time separations for $k=15,30,45,60,75$.

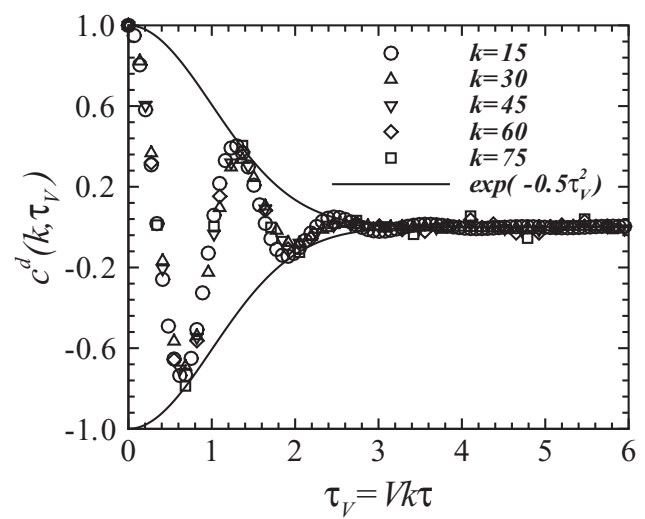

FIG. 4. Normalized time correlations vs normalized time separations $\tau_{V}=V k \tau$ for $k=15,30,45,60,75$.

We can further calculate the space-time correlations of dilatational components using the Fourier transformation of $\hat{R}^{d}(k, \tau)$ from wave-number space to the spatial one:

$$
R^{d}(r, \tau)=\int_{0}^{\infty} \hat{R}^{d}(k, 0) \exp \left(-\frac{1}{2} V^{2} k^{2} \tau^{2}\right) \cos (k \bar{a} \tau) \frac{\sin (k r)}{k r} d k .
$$

Equation (12) can be extended to account for a constant mean velocity. Without loss of generality, the mean velocity is chosen in the direction of $x_{1}$ and denoted as $\left(U_{1}, 0,0\right)$. Applying the coordinate transformation $\left(y_{1}=x_{1}-U_{1} t, y_{2}=x_{2}, y_{3}=x_{3}\right)$ to Eq. (7), we obtain

$$
\begin{aligned}
R^{d}(r, \tau)= & \int_{0}^{\infty} \hat{R}^{d}(k, 0) \exp \left(-\frac{1}{2} V^{2} k^{2} \tau^{2}\right) \\
& \times \cos (k \bar{a} \tau) \frac{\sin \left[k\left(r-U_{1} \tau\right)\right]}{k\left(r-U_{1} \tau\right)} d k .
\end{aligned}
$$

In comparison with the linear wave propagation model, model (13) contains an additional exponential function that is responsible for the random sweeping effect. It also confirms that Taylor's frozen flow model is not a good approximation to the space-time correlation of dilatational components. Wilczek and Narita [26] consider the random sweeping model with constant mean velocity. The present model is consistent with their results.

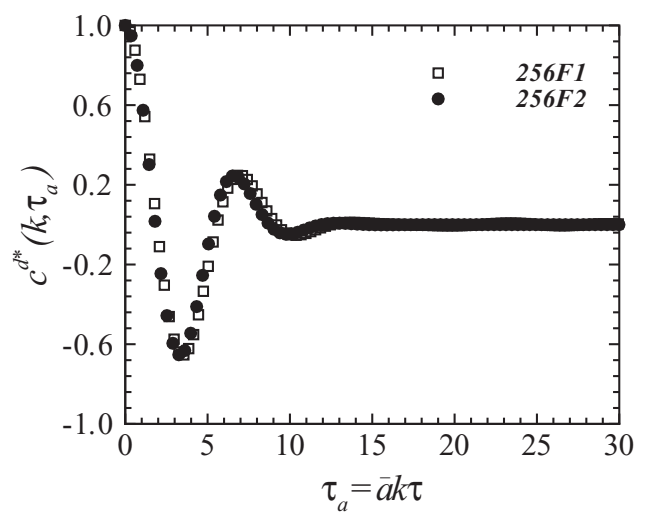

FIG. 5. Rescaled correlation functions vs normalized time separations $\tau_{a}=\bar{a} k \tau$ for $k=15$. 
In summary, we find that solenoidal and dilatational components in compressible isotropic turbulence display different decorrelation processes: a dilatational component is dominated by both random sweeping and wave propagation, while a solenoidal component is dominated by the random sweeping effect. We further develop a swept-wave model for dilatational fluctuations. This model is distinct from the linear wave propagation model since it includes the random sweeping process. The DNS data validate the swept-wave model for compressible isotropic turbulence. Future work is to extend the swept-wave model from compressible isotropic turbulence to turbulent shear flows using the contour approximation approach [27,28].

This work is supported by the National Natural Science Foundation of China under Projects No. 11232011 (Key project) and No. 11021262 (Innovative team) and the National Basic Research Program of China (973 Program) under Project No. 2013CB834100 (Nonlinear science).
[1] R. H. Kraichnan, Phys. Fluids 7, 1723 (1964).

[2] H. Tennekes, J. Fluid Mech. 67, 561 (1975).

[3] V. Yakhot, S. A. Orazag, and Z. S. She, Phys. Fluids A 1, 184 (1989).

[4] S. Y. Chen and R. H. Kraichnain, Phys. Fluids A 1, 2019 (1989).

[5] M. Nelkin and M. Tabor, Phys. Fluids A 2, 81 (1990).

[6] A. A. Praskovsky, E. B. Gledzer, M. Y. Karyakin, and Y. Zhou, J. Fluid Mech. 248, 493 (1993).

[7] T. Sanada and V. Shanmugasundaram, Phys. Fluids A 4, 1245 (1992).

[8] G. W. He, M. Wang, and S. K. Lele, Phys. Fluids 16, 3859 (2004).

[9] R. Rubinstein and Y. Zhou, Phys. Fluids 11, 2288 (1999).

[10] P. Sagaut and C. Cambon, Homogeneous Turbulence Dynamics (Cambridge University Press, Cambridge, 2008).

[11] G. Fauchet and J. P. Bertoglio, C. R. Acad. Sci., Ser. IIb, Mech. Phys. Astron. 327, 665 (1999).

[12] B. Favier, F. S. Godeferd, and C. Cambon, Phys. Fluids 22, 015101 (2010).

[13] Y. Kaneda, T. Ishihara, and K. Gotoh, Phys. Fluids 11, 2154 (1999).
[14] A. Tsinober, P. Vedula, and P. K. Yeung, Phys. Fluids 13, 1974 (2001).

[15] M. Wang, J. B. Freund, and S. K. Lele, Annu. Rev. Fluid Mech. 38, 483 (2006).

[16] R. Benzi, L. Biferale, R. T. Fisher, L. P. Kadanoff, D. Q. Lamb, and F. Toschi, Phys. Rev. Lett. 100, 234503 (2008).

[17] L. Pan, P. Padoan, and A. G. Kritsuk, Phys. Rev. Lett. 102, 034501 (2009).

[18] H. Aluie, Phys. Rev. Lett. 106, 174502 (2011).

[19] G. I. Taylor, Proc. R. Soc. London, Ser. A 164, 476 (1938).

[20] S. Lee, S. K. Lele, and P. Moin, Phys. Fluids A 4, 1521 (1992).

[21] A. Bhagatwala and S. K. Lele, Phys. Fluids 24, 085102 (2012).

[22] D. Stanescu and W. G. Habashi, J. Comput. Phys. 143, 674 (1998).

[23] V. Eswaran and S. B. Pope, Phys. Fluids 31, 506 (1988).

[24] D. T. Gillespie, Phys. Rev. E 54, 2084 (1996).

[25] G. W. He, R. Rubinstein, and L. P. Wang, Phys. Fluids 14, 2186 (2002).

[26] M. Wilczek and Y. Narita, Phys. Rev. E 86, 066308 (2012).

[27] X. Zhao and G. W. He, Phys. Rev. E 79, 046316 (2009).

[28] J. Hogg and G. Ahlers, J. Fluid Mech. 725, 644 (2013). 